\title{
Aplicación de la termodifractometría de neutrones al estudio de procesos de cristalización en mullitas sol-gel.
}

\author{
M.P. VILLAR ${ }^{1}$, L. GAGO-DUPORT ${ }^{2}$, A. HOSER ${ }^{3}$ Y R. GARCÍA ${ }^{1}$ \\ ${ }^{1}$ Dpto. Ciencia de los Materiales e Ingeniería Metalúrgica y Química Inorgánica. Universidad de Cádiz. 11510-Puerto Real (Cádiz). \\ ${ }^{2}$ Dpto. Geociencias Marinas. Universidad de Vigo. 36200-Vigo (Pontevedra).
}

\begin{abstract}
Los experimentos de termodifractometría de neutrones, o difracción de neutrones a alta temperatura, se realizan variando linealmente la temperatura de la muestra con el tiempo. Así, a intervalos de tiempo constantes se obtiene un difractograma que corresponde a un promedio del intervalo de temperaturas variado. Ello permite el seguimiento in-situ de procesos tales como cristalización de amorfos y transiciones de fases en diversos materiales. A pesar de que la mullita es una cerámica ampliamente conocida desde hace años, aún existen aspectos de su formación que no han sido caracterizados de manera bien definida y sobre los que no existe un consenso claro. Uno de ellos es el de la determinación de la secuencia de fases que culminan en su cristalización, sobre todo tratándose de materiales dopados con metales de transición, escasamente estudiados en la literatura. Además, la aplicación de la termodifractometría de neutrones a estos materiales permite eliminar ambigüedades inherentes al empleo de radiación X. Por todo ello, en la presente comunicación, se hace uso de esta técnica para analizar, en tiempo real, el proceso de formación de mullita dopada con $\mathrm{Cr}$ a partir de la cristalización de un gel amorfo sometido a tratamiento térmico en un intervalo de temperaturas comprendido entre 200 y $1400^{\circ} \mathrm{C}$.
\end{abstract}

Palabras clave: difracción de neutrones, mullita dopada con Cr, cristalización, transición de fases

Application of neutron thermodiffractometry to the study of crystallization processes in sol-gel mullites.

Neutron thermodiffractometry experiments are carried out with linear increase of temperature as function of time. At each constant time range a pattern is obtained, corresponding to an average of the temperatures in this interval, so an in-situ study of several processes such as crystallization from amorphous powders and phase transitions is possible. Mullite is a wellknown material, however some controversial concerning aspects of its formation still remains. This is the case of the knowledge about the full phases sequence during mullite crystallization, even more if some transition metal is present in the system. In the present paper we use this techique to analyse, in real time, the Cr-doped mullite formation from an amorphous gel heated in the temperature range of $200-1400^{\circ} \mathrm{C}$.

Keywords: neutron diffraction, Cr-doped mullite, crystallization, phase transition

\section{INTRODUCCIÓN}

La mullita es un aluminosilicato cuya composición se representa habitualmente como $\mathrm{Al}_{2}\left[\mathrm{Al}_{2+\mathrm{x}} \mathrm{Si}_{2-\mathrm{x}}\right] \mathrm{O}_{10-\mathrm{x}^{\prime}}$ donde $x$ se refiere a la fracción de vacantes de oxígeno por fórmula unidad. Esta cerámica puede admitir cantidades variables de ciertos metales de transición $(1,2)$, como $\mathrm{Ti}^{3+}, \mathrm{Ti}^{4+}, \mathrm{V}^{3+}, \mathrm{V}^{4+}, \mathrm{Cr}^{3+}, \mathrm{Mn}^{2+}$, $\mathrm{Mn}^{3+}, \mathrm{Fe}^{2+}, \mathrm{Fe}^{3+} \mathrm{y} \mathrm{Co}^{2+}$, dependiendo, fundamentalmente, del procedimiento empleado para su síntesis y de las condiciones bajo las cuales se desarrolla ésta. La cristalización de mullita a partir de geles amorfos ha sido objeto de estudio en la últimas décadas. Sin embargo, el proceso de transformaciones de fases que conducen al estado final de una cerámica sol-gel no es exclusivamente una función directa de la temperatura final a que se someta el material. Las características iniciales del gel de partida, así como las condiciones de entorno impuestas al sistema para que éste cristalice, son parámetros determinantes de la secuencia de fases que define la pauta general de cristalización de la cerámica. Así, las investigaciones realizadas en este campo durante las últimas décadas indican que los comportamientos frente a la cinética de transformación de fases y densificación observados en sistemas de geles formados por aluminosilicatos dependen, sobre todo, de la escala de mezcla a nivel de precursores en dichos geles. Partiendo de precursores monofásicos, esto es, aquellos que presentan mezcla homogénea a nivel atómico, se consigue formación directa de mullita a temperaturas cercanas a los $980^{\circ} \mathrm{C}$ (3-6); en cambio, la mezcla de precursores difásicos eleva a temperaturas superiores a los $1200^{\circ} \mathrm{C}$ la completa mullitización del material (7-9), previa formación de una fase cúbica tipo espinela o $\gamma-\mathrm{Al}_{2} \mathrm{O}_{3}$.

La adición de un dopante en el sistema aluminosilicato constituye una variable más introducida en el sistema que debe inducir modificaciones apreciables tanto en la pauta de cristalización a mullita como en la estructura última de ésta. Estudios previos apuntan al $\mathrm{Cr}_{2} \mathrm{O}_{3}$ como un agente estabilizador de los cristales de espinela en cerámicas vítreas (10). Rager et al. (11) han estudiado la incorporación de cromo en mullita, estimando que la estructura de mullita puede admitir hasta un $12 \%$ en peso de $\mathrm{Cr}_{2} \mathrm{O}_{3}$, de manera que se produce sustitución de aluminio en huecos octaédricos cuando la adición de $\mathrm{Cr}^{3+}$ tiene lugar en pequeñas cantidades, mientras que a mayores concentraciones de dopante predomina la entrada de $\mathrm{Cr}^{3+}$ en posiciones intersticiales, expandiéndose la red de mullita en el eje c, en mayor medida.

La influencia de la incorporación de cromo en la evolución de los precursores de mullita también ha sido objeto de estudio 
$(12,13)$. En el caso de síntesis de precursores a partir de tetraetilortosilicato (TEOS) y sales de nitrato como aportes de $\mathrm{Al}$ y $\mathrm{Cr}$ se han establecido diferencias en los mecanismos de formación de mullita en función de que el tratamiento térmico impuesto a los precursores sea isotérmico o no isotérmico (12), de manera que en el primero de los casos, la mullita dopada con $\mathrm{Cr}$ es la única fase formada, mientras que en el segundo la formación de mullita a $1100^{\circ} \mathrm{C}$ se ve precedida por la cristalización de una espinela a $910^{\circ} \mathrm{C}$ que coexiste con una mullita rica en $\mathrm{Al}$ y $\mathrm{Cr}$. Por otra parte, la adición de acetato de cromo en solución alcohólica a los precursores promueve la segregación en los mismos de modo que se forman fases intermedias de $\gamma-\mathrm{Al}_{2} \mathrm{O}_{3} \mathrm{y}$ sílice amorfa rica en $\mathrm{Cr}_{2} \mathrm{O}_{3}$ previas a la mullitización (13).

El objetivo del presente trabajo ha sido el análisis, mediante termodifractometría de neutrones, de la secuencia de fases que culmina en la cristalización de mullita durante el tratamiento térmico de sus precursores amorfos, obtenidos mediante síntesis sol-gel y dopados con una determinada cantidad de cromo.

\section{PROCEDIMIENTO EXPERIMENTAL}

\subsection{Materiales e instrumentación}

Las medidas de termodifractometría de neutrones se realizaron en el instrumento E2 del "Berlin Neutron Scattering Center", dotado de un detector sensible a la posición. La longitud de onda viene determinada por el monocromador elegido, en nuestro caso Ge (311), siendo $\lambda$ de 1.21 Å. La colimación del haz se mantuvo en $10^{\prime}$. Los difractogramas, con un rango angular $2 \theta$ comprendido entre 10 y $90^{\circ}$, se registraron in-situ a medida que se producía el calentamiento de los polvos hasta $1400^{\circ} \mathrm{C}$, bajo condiciones de vacío, en el horno de alta temperatura adjunto al difractómetro de polvo. Dicho horno está dotado de un sensor termométrico W-Re (tipo W5) y pantallas de $\mathrm{Nb}$ y $\mathrm{Al}$, con una potencia máxima de calentamiento de 3 kVA que permite alcanzar hasta $1800^{\circ} \mathrm{C}$.

En estos experimentos, los precursores amorfos fueron obtenidos mediante síntesis sol-gel (14), con composición estequiométrica de mullita 3:2 y contenido de $\mathrm{Cr}$ de un $6 \%$ en peso del óxido de dopante. Estos polvos se depositaron en un portamuestras de V, registrando un total de 76 espectros. La menor pendiente en la rampa de temperatura aplicada corresponde al intervalo en el que aparecen fases intermedias, al

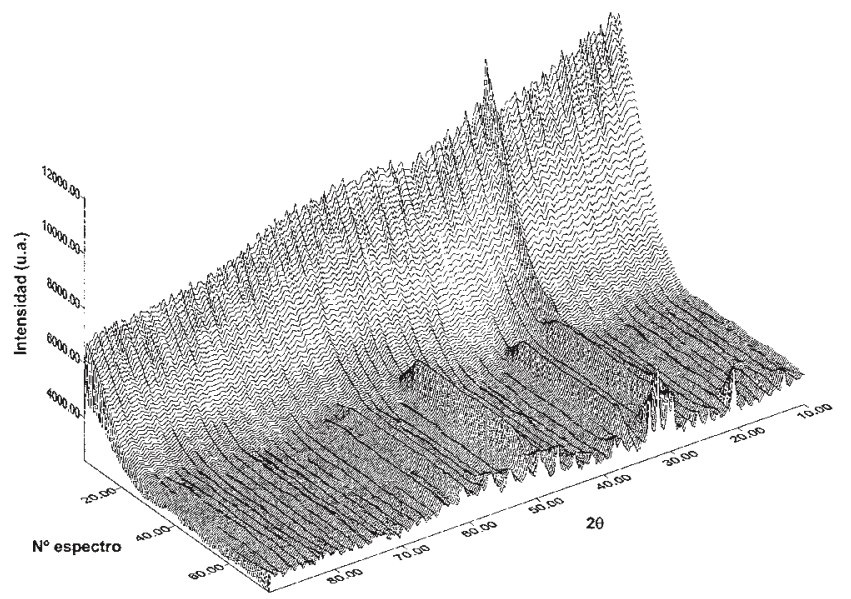

Figura 1. Evolución general del gel inicialmente amorfo durante el proceso de cristalización. objeto de acumular espectros y aumentar así su resolución. Asimismo, para reforzar este efecto en esta región se registraron los difractogramas con un paso angular $2 \theta$ de $0.1^{\circ}$.

\subsection{Tratamiento de datos}

Los espectros, acumulados en intervalos de $20 \mathrm{~min}$, fueron sumados considerando 5 consecutivos con el fin de mejorar la estadística para realizar el análisis cuantitativo. A estos espectros resultantes se les asignó el valor promedio de las temperaturas de los espectros de partida. Por otra parte, a medida que transcurre el tratamiento térmico la muestra sufre cierta contracción debido a una progresiva sinterización que tiene lugar a la vez que cristaliza la mullita. Es necesaria, pues, una corrección de escala de los espectros que permita igualar el número de cuentas en todos ellos.

La cinética de la transformación de fases frente a la temperatura se obtuvo del refinamiento secuencial de los perfiles obtenidos mediante el método de Rietveld, utilizando el programa FullProf (15) a partir de un modelo estructural de varias fases. La reflexión (111) del aluminio, que proviene del horno y aparece en todos los espectros, se incluyó en los refinamientos como fase adicional. La fracción en masa de fase cristalina en relación al amorfo se estimó a partir de la intensidad integrada del fondo de los difractogramas.

\section{RESULTADOS Y DISCUSIÓN}

\subsection{Evolución general del gel: secuencia de cristalización}

El proceso completo de las transformaciones de fases que ocurren durante el tratamiento térmico de los polvos se muestra en las Figuras 1 y 2. La Figura 1 presenta una visión global de la evolución que manifiestan los precursores amorfos durante el tratamiento térmico. La intensidad decrece como resultado de la contracción de la muestra a medida que acontecen fenómenos de sinterización en el material. Dicha contracción ha sido estimada en torno al $60 \%$ en volumen de polvos. En la Figura 2 se muestra el aspecto de los difractogramas después de re-escalados. Las variaciones en la forma del perfil del background podemos asociarlas a la nucleación de fases intermedias, primero, y de la fase mullita, posteriormen-

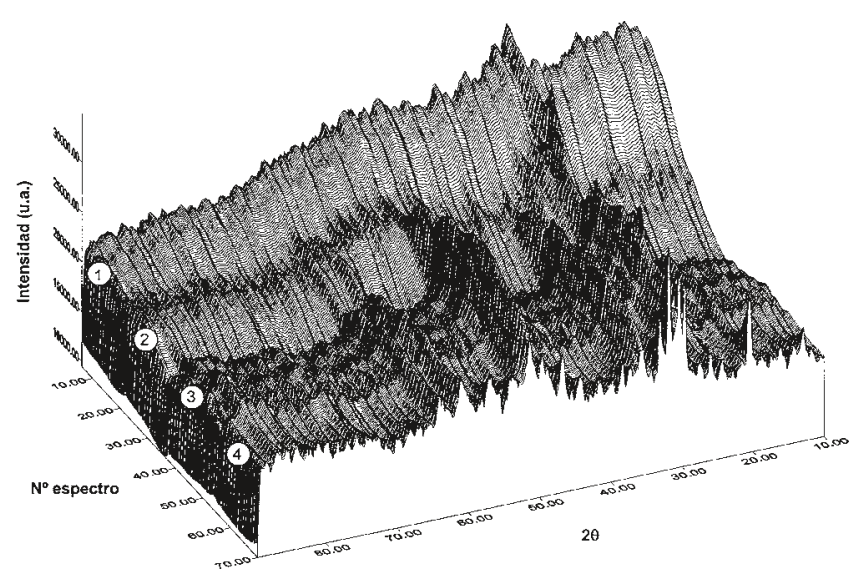

Figura 2. Aspecto de los difractogramas de neutrones normalizados al mismo número total de cuentas mostrando la evolución de las intensidades (proporcional al \% de cada fase) con la temperatura. 


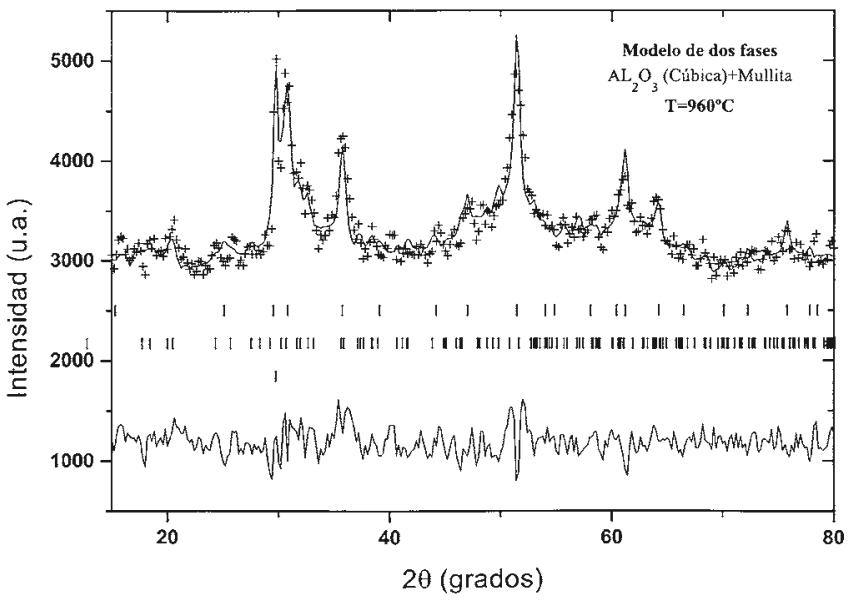

Figura 3. Ajuste Rietveld correspondiente a la suma de espectros de la Región 3, para un modelo estructural de dos fases.

te. De hecho, se pueden establecer cuatro regiones claramente diferenciadas cuyos límites vienen establecidos por dichas variaciones de la forma del perfil del fondo de los espectros:

a) Región 1: intervalo de temperatura ambiente hasta $400^{\circ} \mathrm{C}$. El gel permanece amorfo, siendo la única reflexión presente en estos espectros la correspondiente a la (111) del aluminio del horno.

b) Región 2: intervalo $400-900^{\circ} \mathrm{C}$. Aparecen las primeras reflexiones de una fase cristalina generada a partir del amorfo. Éstas se pueden indexar como una fase tipo espinela, con grupo espacial $\mathrm{Fd} 3 \mathrm{~m}$ y red cúbica de parámetros 7.8-7.9 Å.

c) Región 3: intervalo $900-1100^{\circ} \mathrm{C}$. Además de estas reflexiones, que permanecen, se observa que surgen una serie de picos de poca intensidad que infieren una apariencia rugosa al background de los difractogramas, y que pueden ser indexados a partir de fases de alúmina de simetría tetragonal y/o monoclínica.

d) Región 4: a partir de $1100^{\circ} \mathrm{C}$. Desaparecen tanto las reflexiones de la fase cúbica formada inicialmente como los pequeños picos del background, quedando únicamente las reflexiones de la fase mullita que aumentan en intensidad.

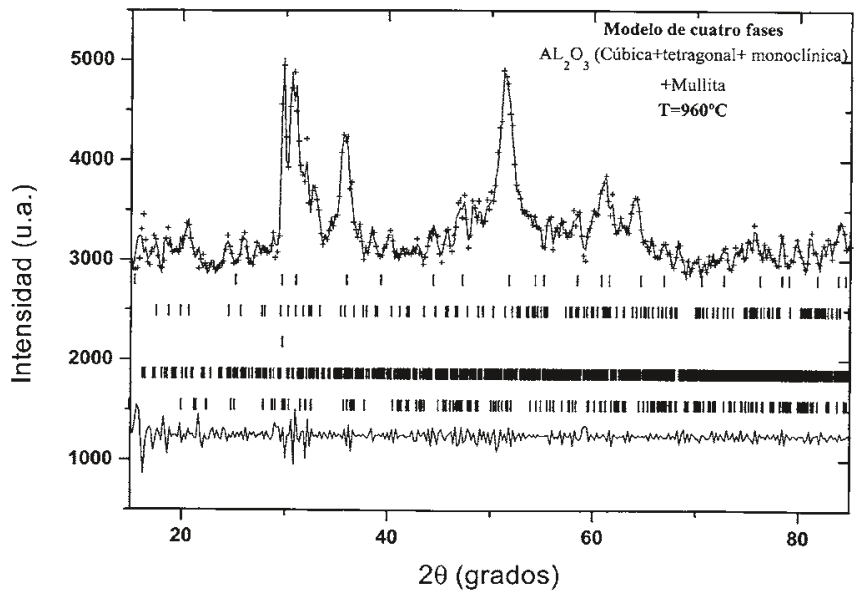

Figura 5. Ajuste Rietveld correspondiente a la suma de espectros de la Región 3, para un modelo estructural de cuatro fases.

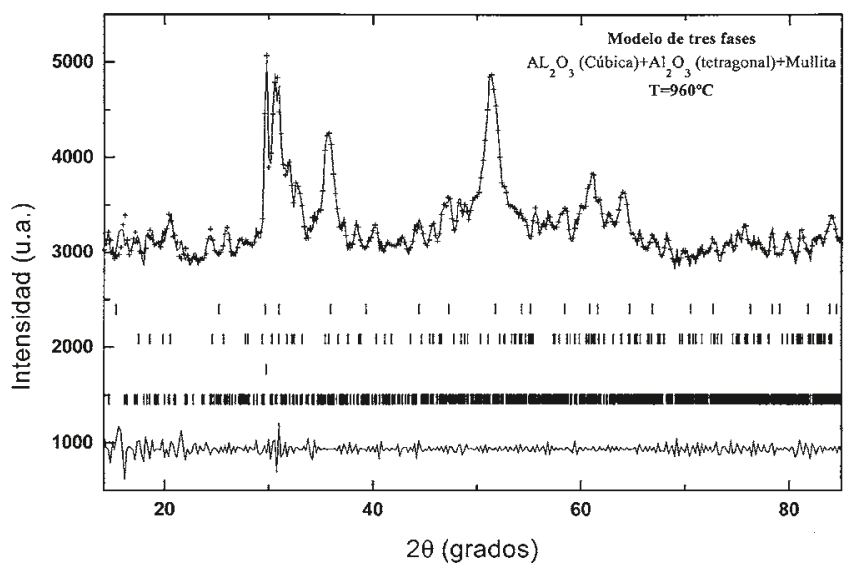

Figura 4. Ajuste Rietveld correspondiente a la suma de espectros de la Región 3, para un modelo estructural de tres fases.

\subsection{Caracterización de las fases intermedias}

Considerando los resultados expuestos en los párrafos anteriores, el modelo estructural multifásico de partida para proceder al refinamiento Rietveld de los espectros de la Región 3 (Figura 2) podría incluir solamente la fase cúbica espinela y la mullita, interpretando los pequeños picos como simples oscilaciones del amorfo, o bien considerar, además, todas aquellas fases de alúmina que contribuyan a la mejora del fondo de los espectros y, consecuentemente, a la disminución del factor de Bragg del ajuste.

En las Figuras 3, 4 y 5 se muestran los ajustes Rietveld a partir de modelos de dos fases (espinela cúbica y mullita), de tres fases (espinela cúbica, alúmina tetragonal y mullita) y de cuatro fases (espinela cúbica, alúmina tetragonal, alúmina monoclínica y mullita), respectivamente. Tanto la alúmina tetragonal como la monoclínica son, de entre todos los polimorfos de alúmina posibles, las únicas fases que disminuyen de manera significativa el factor de Bragg. Estos ajustes se han realizado sobre un espectro promedio que contiene la suma de todos los espectros de esta región. La Tabla I recoge los factores de acuerdo correspondientes a cada ajuste.Como puede apre-

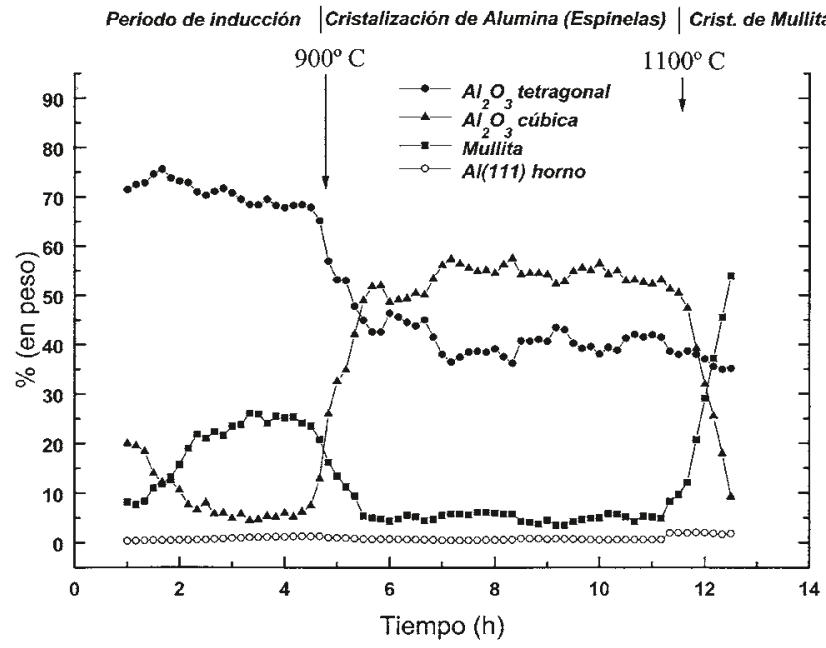

Figura 6. Secuencia de fases del proceso de cristalización en función del tiempo considerando el modelo de dos fases y el de tres fases. 
ciarse, el mejor ajuste se obtiene a partir del modelo trifásico. La Tabla II resume los resultados de la caracterización obtenidos en los ajustes para cada una de las fases consideradas.

\subsection{Cinética y mecanismos de cristalización}

El tratamiento cuantitativo de los datos de difracción mediante Rietveld en el caso de muestras que contengan mezcla de fases cristalinas, como es el sistema que nos ocupa, ha demostrado proporcionar una estimación muy exacta de las cantidades relativas y/o absolutas de cada una de las fases componentes presentes en el sistema (16). Así, partiendo de cada modelo estructural en cuestión, la fracción en peso de cada fase j se ha obtenido a partir de la siguiente relación $(16,17)$ :

$$
W_{j}=S_{j}(Z M V)_{j} / \sum_{i=1}^{n} S_{i}(Z M V)_{i}
$$

donde $W$ es la fracción en peso relativa de cada fase $j$ en una mezcla de $n$ fases, $S$ el factor de escala de Rietveld, $Z$ el número de unidades fórmula por celda unidad, $M$ la masa de la unidad fórmula (en unidades de masa atómica), y $V$ el volumen de la celda unidad (en $\AA^{3}$ ). Por otra parte, la estimación del porcentaje de fase amorfa frente al total de fases cristalinas se ha realizado a partir de la relación entre la intensidad integrada del background y del perfil completo en cada difractograma.

En la Figura 6 se recoge la fracción porcentual de fases cristalinas presentes en función del tiempo teniendo en cuenta los modelos de dos y tres fases, de manera que en todo momento se refiere al $100 \%$ de materia cristalizada. Esta fracción se representa, por otra parte, frente a la temperatura en la Figura 7 únicamente para el modelo de tres fases, aludiendo además al porcentaje de fracción cristalizada con respecto a la fracción de material amorfo.

Como puede inferirse de la Figura 7, en el proceso de cristalización a mullita a partir de sus precursores amorfos dopados con cromo pueden distinguirse tres periodos diferentes, cuyos límites no se modifican sea cual sea el modelo estructural considerado. Así, durante el calentamiento del gel tiene lugar un primer periodo, que denominaremos de inducción, en el cual coexisten pequeñas cantidades de mullita con fases aluminosas. Esto no significa que se consiga una cristalización temprana en el gel, puesto que el nivel de cristalinidad en este intervalo es realmente bajo. Sin embargo, las oscilaciones del background amorfo en los primeros espectros, que por otra parte resultan ser totalmente repetitivas y no aleatorias, como cabría esperar en un amorfo puro, quedan perfectamente ajustadas cuando consideramos estructuralmente mezcla de mullita, espinela cúbica y alúmina tetragonal. Este periodo de inducción claramente finaliza a $900^{\circ} \mathrm{C}$, temperatura a partir de la cual ya es posible afirmar sin género de dudas que la cristalización de las fases origina cristales estructuralmente bien definidos. De hecho, en la Figura 7 se observa cómo el total de amorfo residual se estima aproximadamente en el $25 \%$ en peso. Es la espinela cúbica la fase que porcentualmente experimenta un incremento mayor, a expensas de aquello que estructuralmente asignamos con anterioridad a mullita y a alúmina tetragonal. A partir de $1100^{\circ} \mathrm{C}$ se produce de una manera muy clara la cristalización de mullita y desaparición progresiva de espinela. La Figura 7 permite advertir el hecho de que la disminución del amorfo, tras una cierta estabilización que coincide con el periodo de cristalización de alúminas en la Figura 6, se produce de una manera más suave a partir
TABLA I

FACTORES DE ACUERDO DE LOS REFINAMIENTOS RIETVELD PARA CADA UNO DE LOS MODELOS ESTRUCTURALES: FRACTORES RIETVELD CONVENCIONALES Y FACTORES DE BRAGG PARA CADA FASES.

\begin{tabular}{|c|c|c|c|}
\hline \multicolumn{4}{|c|}{ Modelo de 2 fases } \\
\hline $\mathrm{R}_{\mathrm{p}}=80.9$ & $\mathrm{R}_{\mathrm{wp}}=49.3$ & $\mathrm{R}_{\exp }=22.98$ & $\mathrm{Chi}^{2}=4.61$ \\
\hline \multicolumn{2}{|c|}{$\begin{array}{c}\text { Espinela: } \\
\mathrm{R}_{\text {Bragg }}=32.1\end{array}$} & \multicolumn{2}{|c|}{ Mullita: } \\
\hline$R_{p}=16.1$ & $\mathrm{R}_{\mathrm{wp}}=16.3$ & $\mathrm{R}_{\exp }=15.97$ & $\mathrm{Chi}_{2}=1.04$ \\
\hline $\begin{array}{c}\text { Espinela: } \\
\mathrm{R}_{\text {Bragg }}=5.20 \\
\text { Modelo de } 4 \mathrm{fo}\end{array}$ & $\begin{array}{l}\text { Alúmina tet } \\
\mathrm{R}_{\mathrm{Bragg}}=0\end{array}$ & $\begin{array}{l}\text { tragonal: } \\
.219\end{array}$ & $\begin{array}{l}\text { Mullita: } \\
\mathrm{R}_{\text {Bragg }}: 7.17\end{array}$ \\
\hline $\mathrm{R}_{\mathrm{p}}=12.9$ & $\mathrm{R}_{\mathrm{wp}}=14.6$ & $\mathrm{R}_{\exp }=12.32$ & $\mathrm{Chi}^{2}=1.40$ \\
\hline $\begin{array}{c}\text { Espinela: } \\
\mathrm{R}_{\text {Bragg }}=6.37\end{array}$ & \multicolumn{3}{|c|}{$\begin{array}{ccc}\text { Alúmina tetragonal: } & \text { Alúm. monoclínica: } & \text { Mullita: } \\
\mathrm{R}_{\mathrm{Bragg}}=0.644 & \mathrm{R}_{\mathrm{Bragg}}=12.6 & \mathrm{R}_{\mathrm{Bragg}}=7.00\end{array}$} \\
\hline
\end{tabular}

de esta temperatura. Esto significaría que la mullita se está generando a partir del amorfo, pero al mismo tiempo se disolvería en él la espinela. Es decir, el análisis realizado a partir de los refinamientos de los difractogramas permite sugerir que tiene lugar una transformación mediada por el amorfo resultado de una difusión en estado sólido de Al y Si de la espinela a la fase amorfa, con posterior recristalización en la forma estable mullita. Esta interpretación de los datos concuerda con la idea de que la formación de mullita ocurre mediante mecanismos de nucleación y crecimiento en la matriz amorfa rica en sílice (18), de manera que es precisamente la disolución de las partículas de alúmina en la fase amorfa la que controlaría la velocidad de crecimiento de los granos de mullita $(19,20)$.

El análisis de las Figuras 6 y 7 nos indica que la fracción de alúmina tetragonal, una vez superados $\operatorname{los} 900^{\circ} \mathrm{C}$, se mantiene estable o disminuye muy lentamente. Esto puede explicarse atribuyendo a esta fase un papel de etapa intermedia entre la

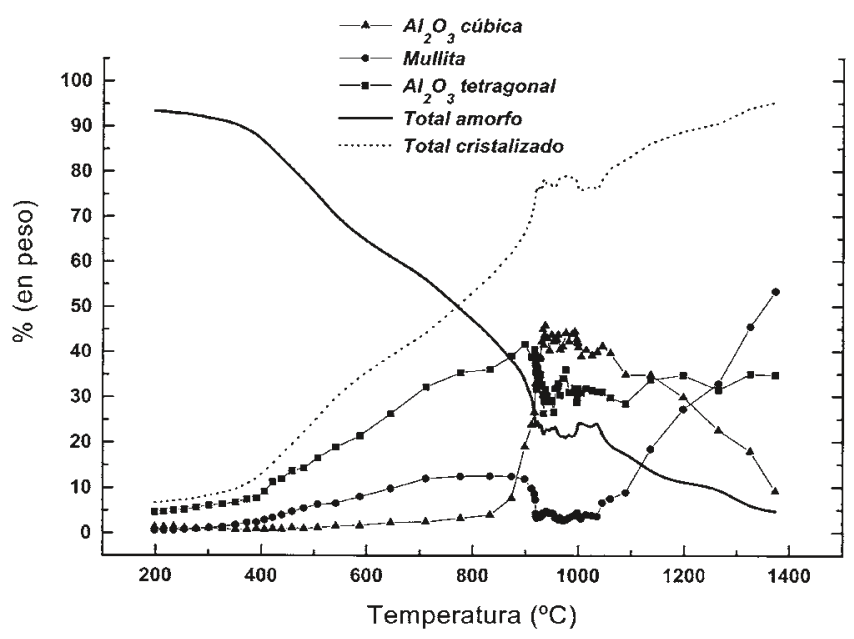

Figura 7. Secuencia de fases del proceso de cristalización en función de la temperatura para el modelo de tres fases, normalizado al porcentaje de fase cristalizada a partir del amorfo. 
formación-desaparición de espinela cúbica y formación de mullita. Atendiendo a los datos de parámetros reticulares de la Tabla 2, una celda tetragonal equivaldría a 3 de espinela, por una parte, y a 9 de mullita, por otra, lo cual refuerza la explicación de la alúmina tetragonal como vehículo entre las fases de espinela y mullita.

\section{CONCLUSIONES}

El estudio mediante difracción de neutrones en tiempo real del proceso de cristalización de un gel amorfo con composición de mullita estequiométrica 3:2 dopado con un 6\% en peso de $\mathrm{Cr}_{2} \mathrm{O}_{3}$ permite concluir que, en este sistema, la formación de mullita no se produce de forma directa a partir de los precursores amorfos. En cambio, tras un periodo inicial de incubación, a partir de $900^{\circ} \mathrm{C}$ cristalizan fases de alúmina distorsionadas precursoras, cuya simetría se ha identificado como cúbica, tetragonal y, en su caso, monoclínica.

La variación que sufre la forma del perfil del background de los espectros a medida que se eleva la temperatura, así como la cuantificación de porcentaje de fase amorfa en cada temperatura, permiten afirmar que el proceso de cristalización de mullita a partir de geles dopados con cromo es un proceso de transformaciones de fase mediados por el amorfo. Proponemos un mecanismo de transformación en el que la fase tetragonal juega un papel de fase intermedia entre la espinela cúbica y la mullita, permitiendo la transición de una a otra.

\section{AGRADECIMIENTOS.}

Los autores agradecen el suministro de muestras a los Dres. Schneider y Saruhan, del DLR (Deutsche Forschungsanstalt für Luft und Raumfahrt, Colonia, Alemania), al BENSC (Berlin Neutron Scattering Center) la aceptación de los experimentos propuestos y la financiación concedida para su ejecución.

\section{BIBLIOGRAFÍA}

1. H. Schneider. "Transition metal distribution in mullite"; p. 135-57. Ceramic Transactions, Vol. 6, Mullite and Mullite Matrix Composites. Editado por S. Somiya, R.F. Davis y J.A. Pask. American Ceramic Society, Westerville, OH, 1990.

2. H. Schneider, K. Okada y J.A. Pask. Mullite and Mullite Ceramics, p. 37-65. John Wiley \& Sons Ltd., Chichester, Reino Unido, 1994.

3. M.J. Hyatt y N.P. Bansal. "Phase transformations in xerogels of mullite composition". J. Mater. Sci., 25, 2815-821 (1990)
TABLA II

GRUPO ESPACIAL Y PARÁMETROS DE RED DE CADA UNA DE LAS FASES CONSIDERADAS:

\begin{tabular}{|lccccccc|}
\hline & \multicolumn{7}{c|}{ Parámetros reticulares } \\
\hline Fase & Grupo espacial & $\mathbf{a}$ & $\mathbf{b}$ & $\mathbf{c}$ & $\alpha$ & $\beta$ & $\gamma$ \\
\hline Espinela & $\mathrm{Fd} 3 \mathrm{~m}$ & 7.8411 & 7.8411 & 7.8411 & 90 & 90 & 90 \\
Alúm. tetragonal & $\overline{\mathrm{P}} \overline{\mathrm{m}} 2$ & 8.0947 & 8.0947 & 25.411 & 90 & 90 & 90 \\
Alúm. monoclínica & $\mathrm{A} 2 / \mathrm{m}$ & 9.3519 & 4.8717 & 5.0249 & 90 & 91.12 & 90 \\
Mullita & $\mathrm{Pbam}$ & 7.4522 & 7.9599 & 2.8495 & 90 & 90 & 90 \\
& & & & & & &
\end{tabular}

4. A.K. Chakravorty y D.K. Ghosh. "Synthesis and $980^{\circ} \mathrm{C}$ phase development of some mullite gels". J. Am. Ceram. Soc., 71 [11], 978-87 (1988).

5. J. Sanz, I. Sobrades, A.L. Cavalieri, P. Pena, S. de Aza y J.S. Moya. "Structural changes induced on mullite precursors by thermal treatment: A ${ }^{27} \mathrm{Al}$ MASNMR investigation". J. Am. Ceram. Soc., 74 [10], 2398-403 (1991).

6. A. Taylor y D. Holland. "The chemical synthesis and crystallization sequence of mullite". J. Non-Cryst. Solids, 12, 1-17 (1993).

7. D.W. Hoffman, R. Roy y S. Komarneni. "Diphasic xerogels, a new class of materials: Phases in the system $\mathrm{Al}_{2} \mathrm{O}_{3}-\mathrm{SiO}_{2}$ ". J. Am. Ceram. Soc., 67 [7], 468-71 (1984)

8. W.C. Wei y J.W. Halloran. "Phase transformation of diphasic aluminosilicate gels". J. Am. Ceram. Soc., 71 [3], 166-72 (1988)

9. D.X. Li y W.J. Thomson, "Mullite formation from nonstoichiometric diphasic precursors". J. Am. Ceram. Soc., 74 [10], 2382-387 (1991).

10. A. Kisilev, R. Reisfeld, A. Buch y M. Ish-Shalom. "Cr(III) in gahnite-containing transparent glass-ceramic: Influence of melting conditions and heat treatment on crystallization and spectroscopic properties". Chem. Phys. Lett., 129 [5], 450-57 (1986).

11. R. Rager, H. Schneider y H. Graetsch. "Chromium incorporation in mullite". Am. Mineral., 75, 392-97 (1990).

12. R. Nass, E. Tkalcec y H. Ivankovic. "Single-phase mullite gels doped with chromium". J. Am. Ceram. Soc., 78 [11], 3097-106 (1995).

13. H. Schneider, K. Ikeda, B. Saruhan y H. Rager. "Electron paramagnetic resonance and optical absorption studies on Cr-doped mullite precursors". J. Eur. Ceram. Soc., 16 [2], 211-15 (1996).

14. H. Schneider, B. Saruhan, D. Voll, L. Merwin y A. Sebald. “Mullite precursor phases". J. Eur. Ceram. Soc., 11, 87-94 (1993).

15. J. Rodríguez-Carvajal. FullProf. Sin publicar.

16. R.J. Hill y C.J. Howard. “Quantitative phase-analysis from neutron powder diffraction data using the Rietveld method". J. Appl. Cryst., 20 [6], 467-74 (1987)

17. R.J. Hill. “Calculated X-Ray powder diffraction data for phases encountered in lead acid battery plates". J. Power Sources, 9 [1], 55-71 (1983)

18. W. Wei y J.W. Halloran. "Transformation kinetics of diphasic aluminosilicate gels". J. Am. Ceram. Soc., 71 [7], 581-87 (1988).

19. S. Sundaresan y I.A. Aksay. "Mullitization of diphasic aluminosilicate gels". J. Am. Ceram. Soc., 74 [10], 2388-392 (1991).

20. C. Gerardin, S. Sundaresan, J. Benziger y A. Navrotsky. “Structural investigation and energetics of mullite formation from sol-gel precursors". Chem. Mater., 6 [2], 160-170 (1994) 